\title{
Guideline pour le diagnostic et le traitement du carcinome intra-canalaire du sein
}

\author{
J.F. Delaloye ${ }^{1,2}$, E. Wight ${ }^{1,2}$, D. Fink ${ }^{1,2}$, R. Otto ${ }^{2,3}$, R. Steiner ${ }^{1,2}$ \\ Groupe de travail «Guideline cancer du sein, carcinome intra-canalaire du sein» *
}

\footnotetext{
1 Société Suisse de Gynécologie et Obstétrique (SSGO)

2 Société Suisse de Sénologie (SSS)

3 Société Suisse de Radiologie (SSR)

* Coordination: E. Eicher (FMH)
}

Correspondance:

Dr J. F. Delaloye

Département de Gynécologie-

Obstétrique CHUV

CH-1011 Lausanne

E-Mail: jean-francois.delaloye@ chuv.hospvd.ch

\section{Résumé}

1. La mammographie est l'examen de choix pour diagnostiquer un carcinome intracanalaire.

2. Une suspicion de carcinome intra-canalaire impose de pratiquer une biopsie stéréotaxique ou chirurgicale.

3. Le diagnostic de carcinome intra-canalaire impose un traitement chirurgical.

4. Le traitement chirurgical consiste soit en une tumorectomie, soit en une mastectomie.

5. La tumorectomie doit passer au large de la lésion, se faire en un seul bloc et être orientée.

6. Le specimen doit être radiographié et comparé à la mammographie de repérage.

7. Une re-excision du site de biopsie s'impose lorsque les tranches de section sont positives.

8. Le spécimen biopsié ne doit pas faire l'objet d'un examen rapide.

9. La mastectomie sous-cutanée ne devrait pas être proposée.

10. La mastectomie controlatérale n'est pas indiquée.

11. Le curage axillaire n'est pas nécessaire pour les petits carcinomes intra-canalaires classés Van Nuys I et II. On peut proposer la recherche du ganglion sentinelle pour les grandes tumeurs et les petites tumeurs classées Van Nuys III.

12. La radiothérapie est recommandée après une tumorectomie.

13. Le tamoxifène peut être recommandé en cas de chirurgie conservatrice.

\section{Introduction}

\section{Relevance}

La généralisation de la mammographie de dépistage entraîne une augmentation du nombre des carcinomes intra-canalaires du sein (de 2.1/ 100000 femmes en 1977-1979 à 9.4/100000 femmes en 1992-1994 dans le Canton de Vaud) [1], qui se présentent le plus souvent comme des lésions non palpables et asymptomatiques.

\section{But}

Ce guide de pratique doit donner aux praticiens des informations et des recommandations pour le diagnostic et le traitement du carcinome intracanalaire du sein.

\section{Méthode}

La FMH a constitué un groupe de travail composé d'experts de différentes spécialités dont la tâche a été d'élaborer ce guide de pratique.

Les recommandations reposent sur une revue systématique de la littérature, répertoriée dans MEDLINE et couvrant la période de 1989 à 2002. Chacune d'elles a été jugée pour son degré de certitude selon la classification de la Canadian Task Force on the Periodic Health Examination [2]; elle se trouve assortie d'un $\varepsilon_{1}, \varepsilon_{2}, \varepsilon_{3}$ ou d'un $\varepsilon_{4}$. Le degré d'évidence $\varepsilon_{5}$, exprimant l'avis consensuel du groupe de travail, n'est pas mentionné. Les recommandations ont été discutées, puis elles ont été testées pendant 3 mois par 60 membres de la FMH avant d'être soumises au comité de la FMH. 


\section{Actualisation}

Ce guide de pratique a une validité de 2 ans. $\mathrm{Au}$ bout de ce laps de temps, il sera réactualisé par le groupe de travail qui l'a élaboré

\section{Diffusion et implémentation}

Ce guide se trouve sur Internet et il est distribué à chaque membre des différentes spécialités concernées. Il servira à la formation pré-graduée, post-graduée et continue.

\section{Financement}

L'élaboration de ces recommandations a été réalisée au sein de la FMH et n'a bénéficié d'aucun support financier.

\section{Base des recommandations}

1. La mammographie est l'examen de choix pour diagnostiquer un carcinome intracanalaire.

La caractéristique radiologique principale du DCIS est la présence d'un groupe de microcalcifications, dont la prise de contraste permet la vision même dans du tissu mammaire dense [3]. Le

\section{Degrés d'évidence \\ (Canadian Task Force on the Periodic Health Examination) [2]}

Degré I: $\quad$ L'évidence repose sur des études randomisées contrôlées (ou métaanalyses) de dimension suffisante pour affirmer que le risque de résultats faussement positifs ou faussement négatifs est minime.

Degré II: L'évidence repose sur des études randomisées contrôlées de dimension trop restreinte pour correspondre à un degré I; elles peuvent montrer une tendance positive, mais non significative statistiquement ou l'absence de tendance. Elles comportent un haut risque de résultats faussement négatifs.

Degré III: L'évidence repose sur des études de contrôles ou de cohortes non randomisées, des séries de cas, des études cas-témoins ou des études transversales.

Degré IV: L'évidence repose sur l'avis d'experts reconnus ou de commissions d'experts exposés dans des conférences de consensus ou sur des recommandations publiées.

Degré V: L'évidence se base sur l'avis des personnes ayant écrit ou actualisé ces recommandations en fonction de leur expérience, de leur connaissance de la littérature se rapportant au sujet et de leurs discussions entre spécialistes.

Les degrés d'évidence des recommandations sont indiqués par un $\varepsilon_{1}, \varepsilon_{2}$, $\varepsilon_{3}$ ou par un $\varepsilon_{4}$. Le degré d'évidence $V$ exprimant l'avis consensuel du groupe de travail, n'est pas mentionné.
DCIS peut se manifester occasionnellement par une image circonscrite dense et non spécifique, sise dans du tissu mammaire dense lui aussi.

Echographiquement le DCIS n'a pas de critères permettant son diagnostic. Les microcalcifications ne se voient souvent pas. Le DCIS prend parfois l'apparence d'une lésion intra-kystique ou provoque un épaississement des canaux galactophores [4].

2. Une suspicion de carcinome intra-canalaire impose de pratiquer une biopsie stéréotaxique ou chirurgicale.

La ponction à l'aiguille fine a une valeur limitée, car elle ne permet que de reconnaître le caractère malin d'une lésion, sans qu'il soit possible de différencier le DCIS du carcinome canalaire invasif par exemple [5].

Seule la micro-biopsie permet de prendre suffisamment de tissu pour qu'un diagnostic histologique soit posé. La méthode diagnostique de choix est représentée par la biopsie stéréotaxique Mammotome ${ }^{\circledR}$ et système $\mathrm{ABBI}^{\circledR}$ (Advanced Breast Biopsy Instrumentation). Ces deux techniques, réalisables sous contrôle radiologique ou échographique, permettent de prélever des cylindres de tissu. Cependant les patientes, dont les seins sont trop petits ou dont les micro-calcifications se trouvent proches de la paroi thoracique, ne peuvent pas bénéficier d'une telle approche [6, 7].

Le(s) spécimen(s) doi(ven)t être radiographié(s), afin de s'assurer qu'il(s) contien(nen)t toutes ou partie des micro-calcifications. Si toutes ces micro-calcifications ont été enlevées, on prendra soin de laisser un clip dans le sein, afin de pouvoir localiser le site de biopsie [6, 7].

Si ces approches diagnostiques ne sont pas réalisables ou si elles ont échoué, on doit procéder à une biopsie à ciel ouvert. Celle-ci requiert un repérage mammographique. Le site à biopsier est repéré soit à l'aide d'un harpon soit à main levée (triangulation) [6, 7].

3. Le diagnostic de carcinome intra-canalaire impose un traitement chirurgical.

On ne peut en effet exclure qu'un carcinome intra-canalaire puisse évoluer en cancer invasif, le risque étant de $32 \%$ à 30 ans pour les lésions de bon pronostic [8: $\left.\varepsilon_{3}\right]$.

4. Le traitement chirurgical consiste soit en une tumorectomie, soit en une mastectomie. 
Tableau 1

Score de Van Nuys.

\begin{tabular}{llll} 
& $\mathbf{1}$ & $\mathbf{2}$ & $\mathbf{3}$ \\
\hline Taille $(\mathrm{mm})$ & $\leq 15$ & $16-40$ & $\geq 41$ \\
\hline Marges $(\mathrm{mm})$ & $\geq 10$ & $1-9$ & $<1$ \\
\hline Grade nucléaire & 1 et 2 & 1 et 2 & 3 \\
\hline & pas de nécrose & nécrose & avec ou sans nécrose
\end{tabular}

La décision de se contenter d'une tumorectomie ou de proposer une mastectomie dépend de la taille de la tumeur, des marges de résection, du degré de différenciation nucléaire et de la dimension du sein. On peut décider de tenir compte du score de Van Nuys (tab. 1) [9]. La taille de la tumeur ne devrait pas excéder 5 centimètres.

Il n'existe pas d'étude randomisée comparant ces deux approches. La survie globale est comparable, que la patiente ait subi un traitement conservateur ou une mastectomie [10-12]. La survie à dix ans après une mastectomie varie de 98 à 100\%, alors que la survie à huit ans après une tumorectomie plus radiothérapie varie de 95 à 100\% [11-13: $\left.\varepsilon_{3}\right]$.

5. La tumorectomie doit passer au large de la lésion, se faire en un seul bloc et être orientée.

L'incision cutanée doit se faire à l'aplomb de la lésion et doit être assez longue pour permettre l'exérèse du spécimen en un seul large fragment [14]. Il a été démontré que le carcinome intra-canalaire peut s'étendre à plus de $2 \mathrm{~cm}$ des zones de micro-calcifications [15]. Le risque de n'avoir pas enlevé toute la tumeur est pourtant relativement faible, lorsque les marges de résection excèdent $10 \mathrm{~mm}$ [16]. L'exérèse en plusieurs fragments empêche une bonne évaluation des tranches de section.

Le fragment doit être orienté. On utilise de préférence des fils, éventuellement marqués par des clips, plutôt que des clips seuls, qui peuvent se rétracter dans le tissu prélevé au moment de la préparation de l'anatomo-pathologiste [7].

On peut éviter de draîner le lit de tumorectomie [6].

6. Le specimen prélevé doit être radiographié et comparé à la mammographie de repérage.

La pièce est radiographiée pendant l'opération, afin de vérifier que toute la lésion suspecte mammographiquement a bien été excisée. La présence de micro-calcifications en bordure du spécimen suggère la présence d'une tumeur résiduelle dans le sein [9]. Une recoupe chirurgicale faite sur le versant suspect (identifiable grâce au marquage du spécimen) est alors indiquée [1].

Si la mammographie du spécimen confirme que tous les éléments suspects ont été enlevés, ceci ne signifie pas que toute la lésion a bien été excisée! On sait en effet que le carcinome intracanalaire peut se développer de manière discontinue [9].

Si la mammographie et/ou l'examen histologique font penser que l'exérèse a été incomplète, une mammographie peut être répétée quatre semaines après la biopsie (éventuellement plus tard si le sein est très tendu et douloureux) [6].

7. Une re-excision du site de biopsie s'impose lorsque les tranches de section sont positives.

Il importe que les tranches de section passent suffisamment au large de la lésion (aucune limite inférieure n'est imposée).

Si l'analyse histologique révèle que les tranches de section passent dans la tumeur, une reprise du lit de tumorectomie s'impose [17].

8. Le spécimen biopsié ne doit faire l'objet d'un examen rapide.

L'examen rapide (extemporané) peut faire manquer un diagnostic de micro-invasion en altérant le spécimen [6].

9. La mastectomie sous-cutanée ne devrait pas être proposée.

Cette opération laisse en place 10 à $15 \%$ de tissu mammaire et n'élimite donc pas complètement le risque de récidive locale $\left[11,18: \varepsilon_{4}\right]$.

10. La mastectomie controlatérale n'est pas indiquée.

L'existence d'un carcinome intra-canalaire contro-latéral est faible. Elle est évaluée à 19\%, ce qui correspond aux 16\% de carcinomes intracanalaires découverts lors d'autopsies faites chez des patientes asymptomatiques [19, 20].

11. Le curage axillaire n'est pas nécessaire pour les petits carcinomes intra-canalaires classés Van Nuys I et II. On peut proposer la recherche du ganglion sentinelle pour les tumeurs étendues et pour les petites tumeurs classées Van Nuys III. 
Tout le monde n'est pas d'accord sur ce point [17]. En cas de tumorectomie pour une petite lésion bien ou moyennement différenciée, il n'est pas nécessaire de soumettre la patiente à un curage axillaire $[6,7]$. Sachant que le risque de métastatisation ganglionnaire varie de $3-4 \%$ à 5-13\% lorsqu'un examen immuno-histochimique est fait pour une lésion classée Van Nuys III (tab. 1) [21, 22: $\left.\varepsilon_{3}\right]$, la recherche du ganglion sentinelle peut trouver sa place en cas de tumorectomie ou de mastectomie pour ce type de lésion [22-25].

\section{La radiothérapie est recommandée après une tumorectomie.}

Il existe des études randomisées (NSABP B-17, EORTC 10853) comparant le devenir des patientes traitées par tumorectomie seule ou par tumorectomie et radiothérapie complémentaire [26-28]. Le risque de récidive locale est plus grand après une tumorectomie seule (16-27\%) qu'après une tumorectomie et une irradiation du sein $(9-12 \%)$ [27-28: $\left.\varepsilon_{1}\right]$. Ce risque dépend notamment des tranches de section chirurgicales [29].

Des études non randomisées ont montré des taux de récidive analoguesaux taux observés dans les études randomisées: $10-17 \%$ pour les patientes traitées par tumorectomie et irradiation et $15-32 \%$ pour les patientes traitées par tumorectomie seule [30-33: $\varepsilon_{3}$ ]. L'irradiation réduit donc le risque, mais ne l'élimine pas complètement $\left[10,34,35: \varepsilon_{3}\right]$.

Des études sont en cours, qui testent la valeur de la radiothérapie pour les tumeurs de bon pronostic. Tant que les résultats ne seront pas connus, nous préconisons une irradiation systématique après une tumorectomie.

\section{Le tamoxifène peut être recommandé en cas de chirurgie conservatrice.}

L'étude randomisée NSABP B-24 a montré que le risque de développer un cancer invasif était significativement plus faible chez les patientes traitées conservativement et recevant du tamoxifène que dans le groupe placebo $(4,1 \%$ versus $7,2 \%, p=0,004)$; le risque de récidive de carcinome in situ était lui aussi abaissé $(4,2 \%$ versus $6,2 \%, p=0,08)\left[36: \varepsilon_{1}\right.$.

\section{Références}

1 Levi F, Te VC, Randimbison L, La Vecchia C. Trends of in situ carcinoma of the breast in Vaud, Switzerland. Eur J Cancer 1997;6:903-6.

2 Clinical practice guidelines for the care and treatment of breast cancer:a Canadian consensus document. CMAJ 1998;158:S3-S8.

3 Stomper PC, Connolly JL, Mayer JE, Harris JR. Clinically occult ductal carcinoma in situ detected with mammography: analysis of 100 cases with radiologic-pathologic correlation. Radiology 1989;172:235-41.

4 Kamio T, Kameoka S. Ultrasonographic diagnosis of intraductal speading of breast cancer: study on nonpalpable breast cancer. Jpn J Breast Cancer 1991;6:455-64.

5 Shin HJ, Sneige N. Is a diagnosis of infiltrating versus in situ ductal carcinoma of the breast possible in fine-needle aspiration specimens? Cancer 1998;84:186-91.

6 Winchester DP, Strom EA. Standards for diagnosis and management of ductal carcinoma in situ (DCIS) of the breast. CA Cancer J Clin 1998; 48:108-28.

7 Clinical practice guidelines for the care and treatment of breast cancer: a Canadian consensus document. CMAJ 1998;158:S27-S34.

8 Page D, Dupont W, Rogers L, Jensen R, Schuyler P. Continued local recurrence of carcinoma 15-25 years after a diagnosis of low grade ductal carcinoma in situ of the breast treated only by biopsy. Cancer 1995;76:1197-200.

9 Silverstein M, Lagios M, Craig P, Waisman J, Colburn W, Levinsky B, et al. A prognostic index for ductal carcinoma in situ of the breast. Cancer 1996;77:2267-74.

10 McCormick B, Rosen PP, Kinne D, Cox L, Yaholam J. Ductal carcinoma in situ of the breast: an analysis of local control after surgery and radiotherapy. Int J Radiat Oncol Biol Phys 1991; 21:289-92.

11 Moore MM. Treatment of ductal carcinoma in situ of the breast. Semin Surg Oncol 1991;7:267-70.

12 White J, Levine A, Gustafson G, Wimbish K, Ingold J, Pettinga J, et al. Outcome and prognosis factors for local recurrence in mammographically detected DCIS of the breast treated by surgery and radiation therapy. Int J Radiat Oncol Biol Phys 1995;31:791-7.

13 Kuske RR, Bean JM, Garcia DM, Perez CA, Andriole D, Philpott G, et al. Breast conservation therapy for intraductal carcinoma of the breast. Int J Radiat Oncol Biol Phys 1993;26:391-6.

14 Silverstein MJ, Gierson ED, Colburn WJ, Cope LM, Furmanski M, Senofsky GM, et al. Can intraductal breast carcinoma be excised completely by local excision? Cancer 1994;73:2985-9.

15 Holland R, Hendricks JH, Verbeek AL, Mravunac M, Schuurmans Stekhoven JH. Extent, distribution, and mammographic/histological correlations of breast carcinoma in situ. Lancet 1990;335:512-22. 
16 Holland R, Faverley DRG. Whole organ studies. In: Silverstein MJ (ed.). Ductal Carcinoma in Situ of the Breast. Baltimore: Williams \& Wilkins; 1997. p. 233-40.

17 Skinner KA, Silverstein MJ. The management of ductal carcinoma in situ of the breast. Endocrine-Related Cancer 2001;8:33-45.

18 Price P, Sinnett HD, Gusterson B, Walsh G, A'Hern RP, McKinna JA. Duct carcinoma in situ: predictors of local recurrence and progression in patients treated by surgery alone. Br J Cancer 1990;61:869-72.

19 Nielsen M, Jensen J, Andersen J. Precancerous and cancerous breast lesions during lifetime and at autopsy: a study of 83 women. Cancer 1984, 54:612-5.

20 Carson W, Sanchez-Forgach E, Stoper P, et al. Lobular carcinoma in situ: observation without surgery as an appropriate therapy. Ann Surg Oncol 1994;1:141-6.

21 Simpson T, Thirlby RC, Dail DH. Surgical treatment of ductal carcinoma in situ of the breast: 10- to 20-year follow-up (review). Arch Surg 1992;127:468-72.

22 Dimpfl T, Genz T, Jung E, et al. Carcinoma in situ der Mamma: ist eine axillare Lymphadenektomie notwendig? Geburtsh u Frauenheilk 1996; $56: 18-22$.

23 Hansen N, Giuliano A. Axillary dissection for ductal carcinoma in situ. In: Silverstein MJ (ed.). Ductal Carcinoma in Situ of the Breast. Baltimore: Williams \& Wilkins; 1997. p 577-84.

24 Silverstein MJ. Ductal carcinoma in situ of the breast: controversial issues. Oncologist 1998; 3:94-103.

25 Klauber-DeMore N, Tan L, Liberman L, Kaptain S, Fey J, Borgen P, et al. Sentinel lymph node biopsy: is it indicated in patients with high-risk ductal carcinoma-in-situ and ductal carcinoma-in-situ with microinvasion? Ann Surg Oncol 2000;7: 636-42.

26 Fisher B, Costantino J, Redmond C, Fisher E, Margolese R, Dimitrov N, et al. Lumpectomy compared with lumpectomy and radiation therapy for the treatment of intraductal breast cancer. N Engl J Med 1993;328:1581-6.

27 Fisher B, Dignam J, Wolmark N, Mamounas E, Costantino J, Poller W, et al. Lumpectomy and radiation therapy for the treatment of intraductal breast cancer: findings for the National Surgical Adjuvant Breast and Bowel Project B-17. J Clin Oncol 1998;16:441-52.
28 Julien J, Bijker N, Fentiman I, Peterse J, Delledonne V, Rouanet P, et al. Radiotherapy in breast conserving treatment for ductal carcinoma in situ: first results of EORTC Randomized Phase III Trial 10853. Lancet 2000;355:528-33.

29 Bijker N, Peterse JL, Duchateau L, Julien JP, Fentiman IS, Duval C, et al. Risk factors for recurrence and metastasis after breast-consering therapy for ductal carcinoma-in-situ: analysis of European Organisation for Research and Treatment of Cancer Trial 10853. J Clin Oncol 2001;19:2263-71.

30 van Zee K, Liberman L, Samli B, Tran K, McCormick B, Petrek J, Rosen P, Borgen P. Long term follow-up of women with ductal carcinoma in situ treated with breast-conserving surgery: the effect of age. Cancer 1999;86:1757-67.

31 Weng E, Juillard G, Parker R, Chang H, Gornbein J. Outcomes and factors impacting local recurrence of ductal carcinoma in situ. Cancer 1999;88: 1643-9.

32 Ringberg A, Idvall I, Ferno M, Anderson H, Anagnostaki L, Boiesen P, et al. Ipsilateral local recurrence in relation to therapy and morphologic characteristics in patients with ductal carcinoma in situ of the breast. Eur J Surg Oncol 2000;26:444-51

33 Cutuli B, Cohen-Solal-Le Nir C, de Lafontan B, Mignotte H, Fichet V, Fay R, et al. Breastconserving therapy for ductal carcinoma in situ of the breast: the French Cancer Centers' experience. Int J Radiat Oncol Biol Phys 2002;53:868-79.

34 Stotter AT, McNeese M, Oswald MJ, Ames FC, Romsdahl MM. The role of limited surgery with irradiation in primary treatment of ductal in situ breast cancer. Int J Radiat Oncol Biol Phys 1990; 18:283-7.

35 Solin LJ, Kurtz J, Fourquet A, Amalric R, Recht A, Bornstein BA, et al. Fifteen-year results of breast conserving surgery and definitive irradiation for the teatment of ductal carcinoma in situ. J Clin Oncol 1996;14:754-63.

36 Fisher B, Dignam J, Wolmark N, Wickerman D, Fisher E, Mamounas E, et al. Tamoxifene in treatment of intraductal breast cancer: National Surgical Adjuvant Breast and Bowel Project B-24 randomized controlled trial. Lancet 1999;353: 1992-2000. 